\title{
Correlation properties of dual polarized antennas with finite pattern orthogonality in mobile fading channels
}

\author{
G. Armbrecht, O. Klemp, and I. Rolfes \\ Leibniz Universität Hannover, Institut für Hochfrequenztechnik und Funksysteme, Appelstr. 9A, 30167 Hannover, Germany
}

\begin{abstract}
Starting from planar broadband log.-per. antenna design, offering the possibility of dual-polarized reception properties, in this article a generalized mathematical approach for rapidly estimating the resulting signal correlation coefficient in a stochastically modeled propagation environment solely based on measured or simulated radiation characteristics of one single antenna element is presented. The obtained results are marking an upper limit and are describing the worst-case scenario according to the signal correlation at the antenna feeding points in terms of line-ofsight (LOS) reception in main beam direction. The knowledge of the derived relationship may be helpful especially for antenna designers to combine antenna performance values with the significant communication system performance parameters, as e.g. in case of Multiple-Input Multiple-Output (MIMO) and diversity configurations.
\end{abstract}

\section{Introduction}

Multiple-Input Multiple-Output (MIMO) and diversity techniques will be an integral part of future mobile communication systems. With the aim of providing higher data rates or decreased outage probabilities, multi element antenna techniques seek for standardization in cellular systems as well as in the domain of wireless networks. Following Jensen and Wallace (2004), the realistic antenna properties as well as multipath channel propagation characteristics play a role in determining the communication performance. As a consequence from space limitations on the mobile side, the application of multimode operating air interfaces offering the capabilities of polarization- and pattern diverse sensing provide a superior usage in comparison to conventional spatial diversity techniques. Our research work is especially focused on four-arm planar log.-per. antennas with two orthogonal polarized excitation modes, as shown in Klemp et al. (2004, 2005b). In accordance with Klemp and Eul (2005) and

Correspondence to: G. Armbrecht

(armbrecht@hft.uni-hannover.de)
Klemp et al. (2005a), systems equipped with such antenna elements show a significantly improved MIMO and diversity system performance. Due to the combination of broadband reception capabilities as well as excellent cross-polarization suppression over a wide frequency range (e.g. 1.7 up to $6.0 \mathrm{GHz}$ ), these structures are promising candidates for the integration in mobile devices and hand-held applications. However, finite pattern orthogonality between the two linear polarized operational modes of a multimode antenna yields an unwanted signal cross-coupling, as depicted in Fig. 1, that represents a limiting factor with respect to diversity gain or MIMO channel capacity.

For that reason we intend to derive a generalized mathematical approach for rapidly estimating the resulting signal correlation coefficient just based on measured or simulated radiation characteristics of one single antenna element. Without loss of generality, the presented theory can be applied to any other dual-polarized antenna configuration supporting two independent operational modes, e.g. the crossdipole. Considering the antenna in a conventional angle-ofarrival (AOA) multipath propagation environment, modeling the AOA dependency in a stochastic formulation according to Pedersen et al. (2000) and Kermoal et al. (2002), we are able to express the modal correlation exclusively in terms of the related field quantities. As a consequence from the symmetry properties of the considered antenna, co- and crosspolar pattern performances can be related to one of the regarded excitation modes. The derived approach will illustrate the relationship of signal-based correlation and finite cross-polarization decoupling between the considered operational modes in a certain line-of-sight (LOS) situation with vanishing angular spreads. This leads to an estimation of a distinct worst-case threshold value for signal-based correlation of dual-polarized operating antennas. Based on these results, antenna designers can easily predict the anticipated upper limit of signal correlation if a distinct antenna design will be applied in a multipath propagation scenario. In this paper, planar four-arm trapezoidal antennas with $M=1$ and $M=5$ periods of the trapezoidal unit cell geometry are utilized to

Published by Copernicus Publications on behalf of the URSI Landesausschuss in der Bundesrepublik Deutschland e.V. 


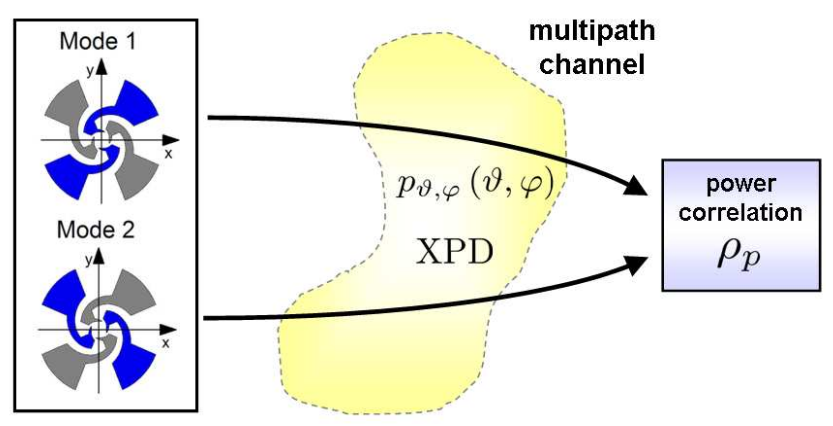

Fig. 1. Finite pattern orthogonality of a dual-polarized trapezoidal antenna $(M=1)$ in a multipath propagation scenario yielding unwanted signal correlation.

validate the derived formulation by comparing the theoretical correlation limits with the antenna cross-polarization suppression obtained from the simulated radiation pattern. See Armbrecht et al. (2006) for detailed information about the two trapezoidal antennas.

\section{Antenna correlation in mobile fading channels}

Multi-port antenna configurations providing two linear excitation modes with finite pattern orthogonality exhibit interelement correlation, which is strongly affected by the properties of the considered multipath propagation environment. A high amount of signal correlation at the antenna feeding point reduces the derived MIMO and diversity system performance significantly. In accordance with Svantesson (2002) the power correlation coefficient $\rho_{\mathrm{p}}$ can be generally computed, as follows:

$\rho_{\mathrm{p}} \approx\left|\rho_{12}\right|^{2}=\left|\frac{R_{12}}{\sqrt{\sigma_{1}^{2} \cdot \sigma_{2}^{2}}}\right|^{2}$,

where $R_{12}$ and $\sigma_{1,2}^{2}$ denote the antenna covariance and variance, respectively. In Taga (1990) the spatial channel properties of a multi element antenna configuration are determined by a two-dimensional probability distribution $p_{\vartheta, \varphi}(\vartheta, \varphi)$ for the AOA behavior at the reception antenna elements in the direction of the elevation angle $\vartheta$ and the azimuth angle $\varphi$. Using the complete antenna far-field representation given by the complex vector components $E_{\vartheta}(\vartheta, \varphi)$ and $E_{\varphi}(\vartheta, \varphi)$ and the proposed two-dimensional probability distribution $p_{\vartheta, \varphi}$, the covariance $R_{12}$ is calculated, as follows:

$$
\begin{aligned}
& R_{12}=\mathrm{K} \int_{0}^{2 \pi} \int_{0}^{\pi}\left[E_{\vartheta, 1}(\vartheta, \varphi) E_{\vartheta, 2}^{*}(\vartheta, \varphi)+\mathrm{XPD} .\right. \\
& \left.E_{\varphi, 1}(\vartheta, \varphi) E_{\varphi, 2}^{*}(\vartheta, \varphi)\right] \cdot p_{\vartheta, \varphi}(\vartheta, \varphi) \cdot \sin \vartheta d \vartheta d \varphi .
\end{aligned}
$$

In our case, the indices 1 and 2 represent the excitation modes of the dual-linear polarized antenna structure. $K$ is

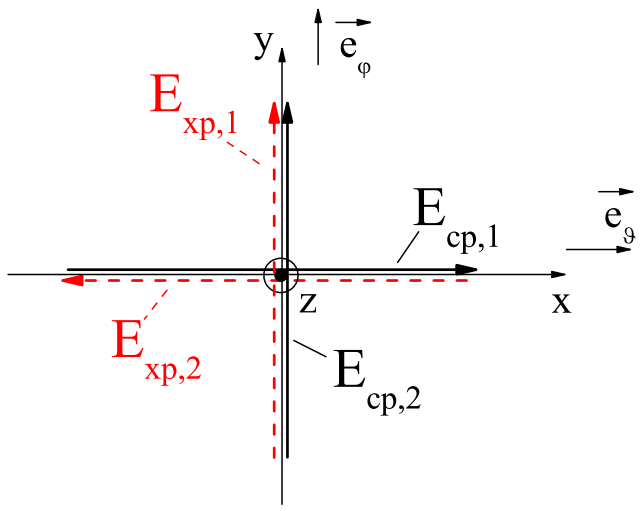

Fig. 2. Far-field dependency in main beam direction $\left(\vartheta_{0}=0^{\circ}\right)$ of a dual-polarized trapezoidal antenna located in the xy coordinate plane.

a proportionality constant and $\left(^{*}\right)$ accounts for the complex conjugate operation. Following Taga (1990), the value of XPD in Eq. (2) denotes the cross polarization discrimination which specifies the ratio of the mean incident power in cross-polarized direction at the receiver to the mean incident power in horizontal direction. The respective variances $\sigma_{1,2}^{2}$ are given by Eq. (3) with $\nu=1,2$.

$$
\begin{aligned}
\sigma_{v}^{2}=\mathrm{K} & \int_{0}^{2 \pi} \int_{0}^{\pi}\left[\left|E_{\vartheta, v}(\vartheta, \varphi)\right|^{2}+\mathrm{XPD} .\right. \\
& \left.\left|E_{\varphi, v}(\vartheta, \varphi)\right|^{2}\right] \cdot p_{\vartheta, \varphi}(\vartheta, \varphi) \cdot \sin \vartheta d \vartheta d \varphi .
\end{aligned}
$$

Spatial separation between the regarded antenna elements can be neglected due to the fact that the phase centers of all antenna elements coincide in case of polarization diverse sensing.

2.1 Line-of-sight (LOS) propagation scenario with deterministic impinging waves at the receiver

To draw a relationship between the correlation properties and the field-based quantities of the antenna elements, it is necessary to assume a line-of-sight scenario featuring deterministic impinging wave propagation at the reception side. As given by Eq. (4), this behavior can easily be described in compliance with Fujimoto and James (2000) by modifying the stochastic channel description using delta distributions in $\vartheta$ - and $\varphi$-direction.

$p_{\vartheta, \varphi}(\vartheta, \varphi)=\frac{\delta\left(\vartheta-\vartheta_{0}\right) \cdot \delta\left(\varphi-\varphi_{0}\right)}{\sin \vartheta}$

Substituting $p_{\vartheta, \varphi}(\vartheta, \varphi)$ in Eq. (2) with Eq. (4), the delta distributions will directly fade out the complex field quantities of the two adjacent antenna elements in the direction defined by the angles $\vartheta_{0}$ and $\varphi_{0}$.

In this article, we assume a deterministic wave distribution impinging from the positive z-direction at $\vartheta_{0}=\varphi_{0}=0^{\circ}$, which 


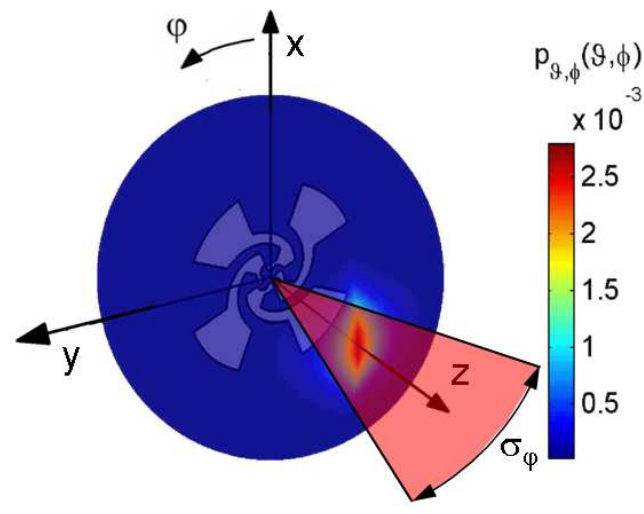

Fig. 3. Variation of the azimuthal angular spread $\sigma_{\varphi}$ in main beam direction of a log.-per. trapezoidal antenna geometry $(M=1)$ located in the xy coordinate plane.

corresponds to the main beam direction of the dual-polarized planar antenna structures located in the xy coordinate plane. In addition, as depicted in Fig. 2, the far-field components of both excitation states can be expressed by considering solely the field quantities of the first mode and representing the fields of the second orthogonal mode by performing a circular permutation. This fact yields to a major simplification according to the mathematical complexity of the aspired formulation. The equivalences accounting for the far-field components of both excitation modes in co- (cp) as well as in cross-polarization $(\mathrm{xp})$ can be formulated, as follows:

Mode 1: $E_{\mathrm{cp}, 1}=E_{\vartheta} ; E_{\mathrm{xp}, 1}=E_{\varphi}$

Mode 2: $E_{\mathrm{cp}, 2}=E_{\varphi} ; \quad E_{\mathrm{xp}, 2}=-E_{\vartheta}$.

2.2 Analytical computation and validation of signal correlation based on far-field quantities

Applying the LOS channel scenario model in combination with the distinct permutation of field components in main beam direction, as introduced in Sect. 2.1, to Eq. (1), a novel analytical approach for the power correlation coefficient $\rho_{\mathrm{p}}^{\mathrm{LOS}}$ is derived, as follows:

$$
\rho_{\mathrm{p}}^{\mathrm{LOS}}=\left|\frac{E_{\vartheta} E_{\varphi}^{*}+\mathrm{XPD} \cdot E_{\varphi}\left(-E_{\vartheta}^{*}\right)}{\sqrt{\left(\left|E_{\vartheta}\right|^{2}+\mathrm{XPD} \cdot\left|E_{\varphi}\right|^{2}\right)\left(\left|E_{\varphi}\right|^{2}+\mathrm{XPD} \cdot\left|E_{\vartheta}\right|^{2}\right)}}\right|^{2} .
$$

Solely depending on complex vector components $E_{\vartheta}$ and $E_{\varphi}$ in main beam direction at $\vartheta_{0}=\varphi_{0}=0^{\circ}$ and the cross polarization discrimination XPD, this equation establishes a direct coupling between field-based and channel-environmentbased quantities. Furthermore, it provides a distinct threshold value for the correlation coefficient marking an upper limit due to the vanishing angular spread of the assumed lineof-sight reception scenario. In compliance with Fujimoto

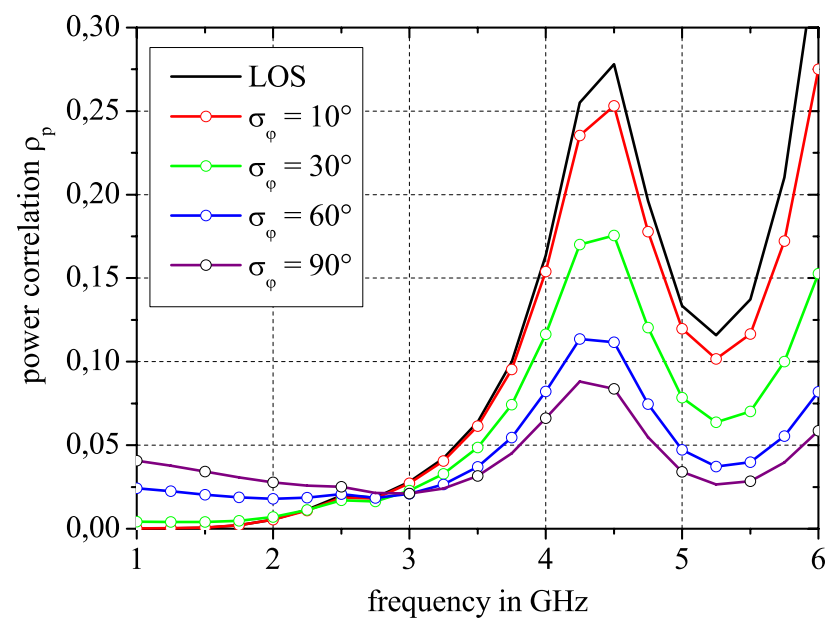

Fig. 4. Validating the proposed analytical correlation threshold value by decreasing the azimuthal angular spread $\sigma_{\varphi} \rightarrow 0$ for a log.per. trapezoidal antenna $(M=1)$.

and James (2000) increasing angular spreads in the stochastically modeled radio channel leads to a decorrelation of the received signals. This behavior is exploited to validate the derived formulation, as depicted in Fig. 3. Decreasing the azimuthal angular spread $\sigma_{\varphi}$ successively must lead to increasing values of the derived power correlation coefficient $\rho_{\mathrm{p}}$ according to Eq. (1) passing into the threshold value $\rho_{\mathrm{p}}^{\mathrm{LOS}}$ for the special case of $\sigma_{\varphi} \rightarrow 0$, as given by Eq. (6). Without loss of generality, the validation process is conducted using one of the introduced log.-per. trapezoidal antenna structures with $M=1$ periods of the trapezoidal unit cell geometry. In Fig. 4, the power correlation coefficient $\rho_{\mathrm{p}}$ is shown over the frequency sweep from 1.0 to $6.0 \mathrm{GHz}$ for various values of the angular spread $\sigma_{\varphi}$ in a range from $90^{\circ}$ down to $10^{\circ}$. In terms of decreasing angular spreads the depicted curves continuously approach the analytical line-of-sight power correlation $\rho_{\mathrm{p}}^{\mathrm{LOS}}$. The obtained approximation verifies the equation given by Eq. (6) yielding a distinct limiting value for vanishing angular spreads in the stochastic channel model for a constant value of $\mathrm{XPD}=0 \mathrm{~dB}$. Subsequently in Sect. 3, the derived equation given by Eq. (6) will be evaluated under the assumption of mitigating phase influences leading to a straightforward relationship between antenna-based and mobile fading channel effects.

\section{Field-based estimation of the correlation performance}

If a choice has to be made among a variety of different antenna structures for the application in wireless communication systems exploiting the multiple propagation path using polarization diverse sensing, always the antenna exhibiting the best performance according to the suppression of the cross-polarized field component will be selected. 


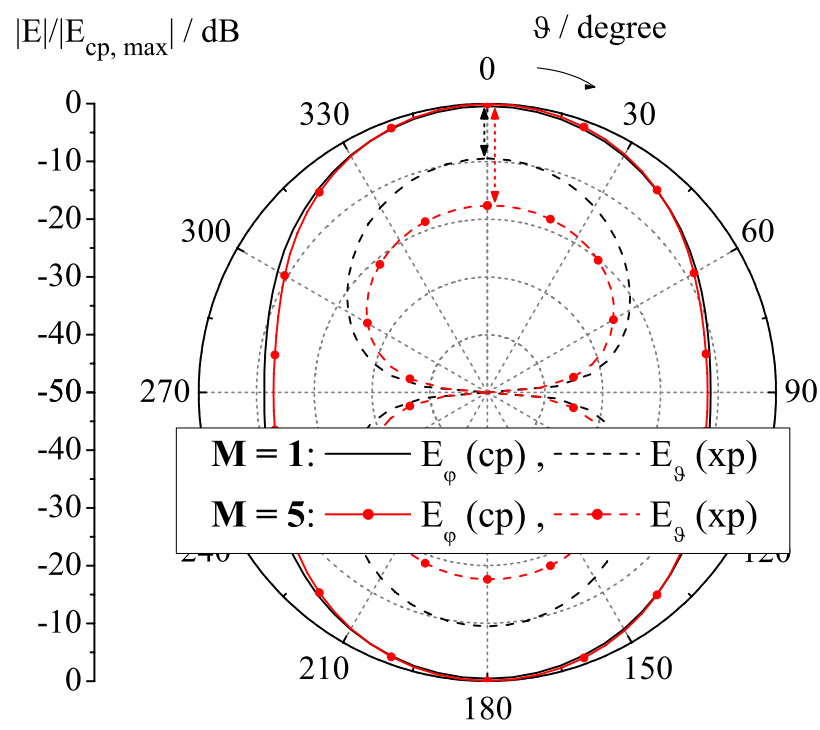

Fig. 5. Simulated far-field radiation pattern of the trapezoidal antennas with $M=1,5$ in free space at $f=4.5 \mathrm{GHz}$ exhibiting different value of XP-suppression in main beam direction at $\vartheta=0^{\circ}$.

Table 1. Upper bound correlation coefficient $\rho_{\mathrm{p}}^{\max }$ derived from ratio of cross polarization suppression $\left|E_{\mathrm{cp}}\right|\left|E_{\mathrm{xp}}\right|$ for two trapezoidal antennas at $f=4.5 \mathrm{GHz}$ compared to correlation values of different scenarios.

\begin{tabular}{cccccc}
\hline & $\left|E_{\mathrm{cp}}\right| /\left|E_{\mathrm{xp}}\right|$ & $\rho_{\mathrm{p}}^{\max }$ & $\rho_{\mathrm{p}}^{\mathrm{LOS}}$ & $\rho_{\mathrm{p}}\left(\sigma_{\varphi}=10^{\circ}\right)$ & $\rho_{\mathrm{p}}\left(\sigma_{\varphi}=60^{\circ}\right)$ \\
\hline$M=1$ & $10 \mathrm{~dB}$ & 0.32 & 0.278 & 0.25 & 0.112 \\
$M=5$ & $18 \mathrm{~dB}$ & 0.05 & 0.014 & 0.01 & 0.008 \\
\hline
\end{tabular}

Disregarding the phase information with respect to the complex vector components $E_{\vartheta}$ and $E_{\varphi}$ in Eq. (6) will lead to a distinct relationship between cross polarization ratio $\left|E_{\mathrm{cp}}\right| /\left|E_{\mathrm{xp}}\right|$ obtained from a radiation pattern representation of the vector field components, as depicted in Fig. 5 for the two considered trapezoidal antennas with $M=1$ and $M=5$ at $f=4.5 \mathrm{GHz}$. Accounting for an in-phase superposition of the co- and cross-polarized field quantities yields to an absolute maximum of the power correlation coefficient $\rho_{\mathrm{p}}^{\max }$ in lineof-sight wave propagation in main beam direction. Hence, in Fig. 6 these upper bound representation of the power correlation $\rho_{\mathrm{p}}^{\max }$ is evaluated in detail concerning various values of the ratio of XP-suppression $\left|E_{\mathrm{cp}}\right| /\left|E_{\mathrm{xp}}\right|$ ranging from $0 \mathrm{~dB}$ to $20 \mathrm{~dB}$ as well as the channel parameter XPD varying from $-30 \mathrm{~dB}$ up to $0 \mathrm{~dB}$.

As depicted, for low value of XPD the proposed correlation coefficient $\rho_{\mathrm{p}}^{\max }$ is close to $\approx 1$ due to the power imbalances according to the perceived power in suppressed polarization state. Actually, this kind of correlation behavior is valid for almost any state of cross polarization ratio. The obtained threshold values of $\rho_{\mathrm{p}}^{\max }$ will be stronger influenced for increasing XPD ratios.

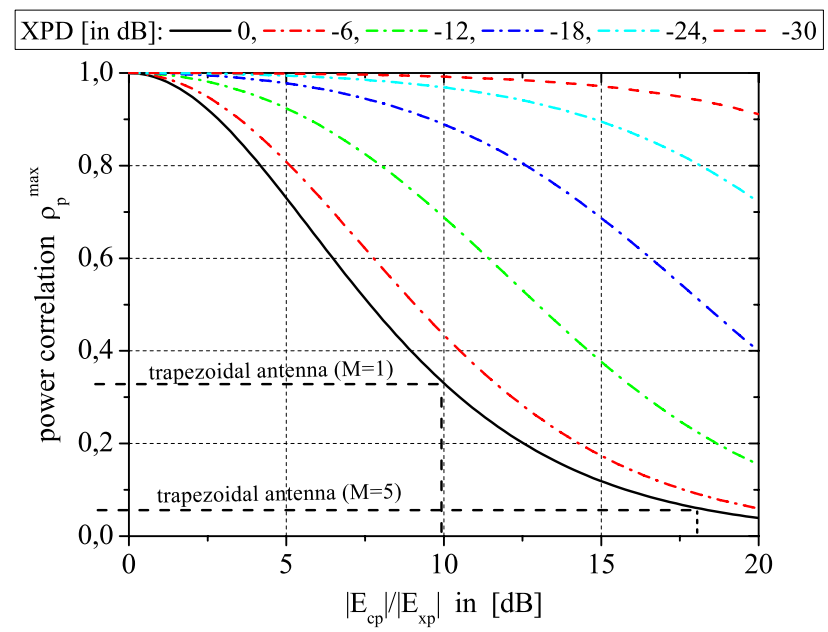

Fig. 6. Maximal correlation behavior $\rho_{\mathrm{p}}^{\max }$ solely depending on the ratio of co- to cross-polarization suppression $\left|E_{\mathrm{cp}}\right| /\left|E_{\mathrm{xp}}\right|$.

As shown in Table 1 for the particular case of $\mathrm{XPD}=0 \mathrm{~dB}$, the correlation effects for $\rho_{\mathrm{p}}^{\max }$ according to Fig. 6 for two trapezoidal antennas at $f=4.5 \mathrm{GHz}$ are verified for the cross polarization ratio extracted from Fig. 5. This novel kind of correlation computation is solely depending on simulated radiation pattern for one of the dual-polarized antenna modes. Furthermore, regarding the LOS representation $\rho_{\mathrm{p}}^{\mathrm{LOS}}$ and the respective angular spreads of $\sigma_{\varphi}=10^{\circ}$ and $60^{\circ}$ representing typical outdoor and indoor scenarios, the threshold value $\rho_{\mathrm{p}}^{\max }$ is proven to be a promising approximation for the worst-case correlation behavior due to the decorrelating impact of wider angular spreads and field-based phase considerations.

\section{Conclusions}

In this paper, a novel approach to effectively estimate the anticipated correlation properties for dual-linear polarized antenna elements in the presence of finite pattern orthogonality is introduced. Depending on simulated or measured radiation patterns, the phase information of the respective antenna configuration can be neglected in order to perform a worstcase estimation for a line-of-sight spatial propagation scenario. Hence, the proposed computation determines an upper threshold value for the correlation coefficient. According to realistic mobile fading channels, the obtained signal correlation will be permanently smaller due to decorrelation effects of a wider angular spread and actual phase properties of the antenna far-field components. The presented investigation offers communication system designers as well as antenna engineers the possibility to predict rapidly the anticipated system performance by solely knowing the utilized antenna radiation pattern. 


\section{References}

Armbrecht, G., Klemp, O., and Eul, H.: Spherical mode analysis of planar frequency-independent multi-arm antennas based on its surface current distribution, Adv. Radio Sci., 4, 25-32, 2006, http://www.adv-radio-sci.net/4/25/2006/.

Fujimoto, K. and James, J. R.: Mobile antenna systems handbook, 2nd Edition, Artech House antennas and propagation library, Boston, London, 2000.

Jensen, M. A. and Wallace, J. W.: A Review of Antennas and Propagation in MIMO Wireless Communications, 52, 2810-2824, 2004.

Kermoal, J. P., Schumacher, L., Pedersen, K. I., Morgensen, P. E., and Frederiksen, F.: A Stochastic MIMO Radio Channel Model With Experimental Validation, 20, 1211-1226, 2002.

Klemp, O. and Eul, H.: On the MIMO capacity of planar, log.per. four-arm antennas, The European Conference on Wireless Technology, ECWT, 2005.
Klemp, O., Schultz, M., and Eul, H.: Miniaturization Techniques For Logarithmically-Periodic Planar Antennas, IEEE 15th International Symposium on Personal, Indoor and Mobile Radio Communications, PIMRC, 2004.

Klemp, O., Schmitz, O., and Eul, H.: Polarization diversity analysis of dual-polarized log.-per. planar antennas, IEEE 16th International Symposium on Personal, Indoor and Mobile Radio Communications, PIMRC, 2005a.

Klemp, O., Schultz, M., and Eul, H.: Novel logarithmically periodic planar antennas for broadband polarization diversity reception, Int. J. Electron. Commun. (AEUE), 59, 268-277, 2005 b.

Pedersen, K. I., Andersen, J., Kermoal, J. P., and Morgensen, P.: A Stochastic Multiple-Input-Multiple-Output Radio Channel Model for Evaluation of Space-Time Coding Algorithms, VTC 2000 Boston, 1, 893-897, 2000.

Svantesson, T.: Correlation and Channel Capacity of MIMO Systems Employing Multimode Antennas, 51, 1304-1312, 2002.

Taga, T.: Analysis for Mean Effective Gain of Mobile Antennas in Land Mobile Radio Environments, 39, 117-131, 1990. 